\title{
СИНДРОМОМ ПОЛИКИСТОЗНЫХ ЯИЧНИКОВ И ИХ ВЗАИМОСВЯЗЬ С МИКРОБИОТОЙ КИШЕЧНИКА И ВЛАГАЛИЩА (ОБЗОР ЛИТЕРАТУРЫ)
}

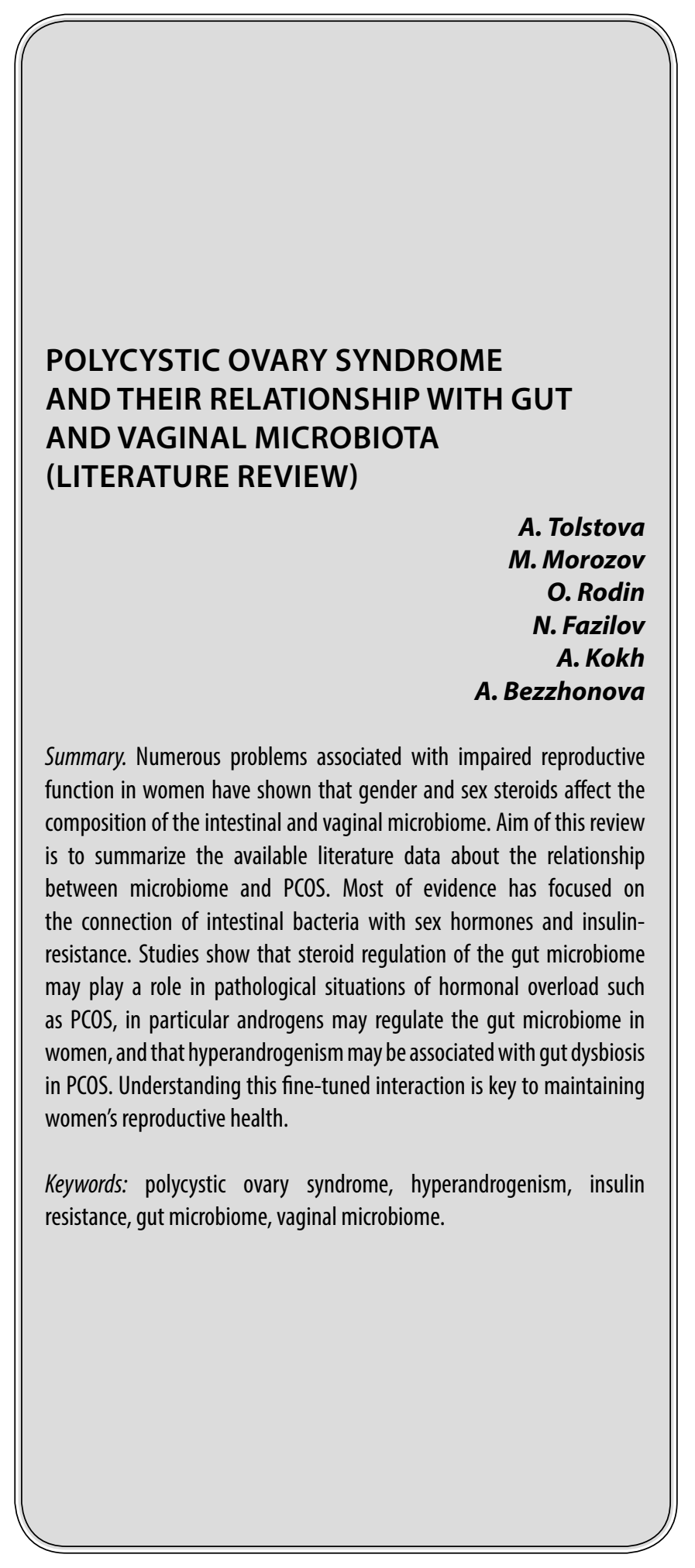

Толстова Анастасия Сергеевна

ФГБОУВО «Национальный исследовательский Мордовский государственный университет им. Н.П. Огарёва» asyatol97@mail.ru

Морозов Максим Владимирович

ФГБОУВО «Национальный исследовательский Мордовский государственный университет им. Н.П. Огарёва» morozov_mv93@mail.ru Родин Олег Васильевич

ФГБОУВО «Начиональный исследовательский Мордовский государственный университет им. Н.П. Огарёва» rodin2oleg@gmail.com

Фазилов Никита Александрович ФГБОУ ВО «Национальный исследовательский Мордовский государственный университет им. Н.П. Огарёва»

Fazilovnikita13@gmail.com

Кох Андрей Александрович

ФГБОУ ВО «Национальный исследовательский Мордовский государственный университет им. Н.П. Огарёва» koh-andrei63@yandex.ru

Безжонова Ангелина Александровна ФГБОУ ВО «Национальный исследовательский Мордовский государственный университет им. Н.П. Огарёва» abezzhonova@mail.ru

Аннотация. Многочисленные проблемы, связанные с нарушением репродуктивной функции женщины, показали, что пол и половые стероиды влияют на состав микробиома кишечника и влагалища. Цель этого обзора - обобщить имеющиеся литературные данные о взаимосвязи между микробиомом и СПКЯ. Большинство доказательств было сосредоточено на связи кишечных бактерий с половыми гормонами и инсулинорезистентностью. Исследования показывают, что стероидная регуляция микробиома кишечника может играть роль в патологических ситуациях гормонального избытка, таких как СПКЯ, в частности андрогены могут регулировать микробиом кишечника у женщин и что гиперандрогения может быть связана с дисбактериозом кишечника при СПКЯ. Понимание этого тонко настроенного взаимодействия является ключом к сохранению репродуктивного здоровья женщин.

Ключевые слова: синдром поликистозных яичников, гиперандрогения, инсулинорезистентность, микробиом кишечника, микробиом влагалища. 


\section{Ввемение}

C индром поликистозных яичников (СПКЯ) является наиболее частой причиной женского эндокринного бесплодия с высокой гетерогенностью, характеризующейся повышенным биосинтезом андрогенов яичниками, ановуляцией [1, 2]; тем не менее, СПКЯ влияет на некоторые аспекты общего здоровья женщин, с долгосрочными последствиями, выходящими далеко за пределы репродуктивного возраста $[3,4,5]$. Распространенность СПКЯ варьируется в зависимости от исследуемой популяции и используемых диагностических критериев и составляет от 8\% до 13\% [6].

Гиперандрогенизм (ГА) - одно из основных расстройств, которые могут характеризовать СПКЯ. Его чрезмерное производство яичниками или корой надпочечников приводит к дерматологическим нарушениям (акне, гирсутизм, андрогенная алопеция), изменению оси гипоталамус-гипофиз-гонад и нарушению регуляции фолликулогенеза яичников, что приводит к нарушению менструального цикла и бесплодию [6]. Согласно Европейскому обществу репродукции человека и эмбриологии СПКЯ определяется наличием 2 из следующих 3 признаков: олиго - и / или ановуляция, клинические и / или биохимические признаки гиперандрогения и / или морфология поликистозных яичников. Наличие 12 или более фолликулов размером 2-9 мм во всем яичнике или объем яичника $=10 \mathrm{~cm}^{3}[8,9]$.

Этиология СПКЯ до сих пор не ясна; однако было выявлено несколько факторов, участвующих в создании гормонального и метаболического дисбаланса, которые могут привести к развитию этого синдрома $[9,10,11]$. Наиболее авторитетной является теория Фрэнка, который считает СПКЯ генетически детерминированным заболеванием, клиническая и биохимическая гетерогенность которого зависит от взаимодействия генетических факторов и факторов окружающей среды [12]. В этиологических гипотезах, выдвинутых на протяжении многих лет, сыграл свою роль тот факт, что помимо гинекологических проблем, СПКЯ может также приводить к системным метаболическим нарушениям (таким как гиперинсулинемия и инсулинорезистентность (ИР), ожирение, повышенный риск диабета II типа, сердечно-сосудистые заболевания). Несмотря на связь СПКЯ с ожирением, мало доказательств того, что диетические факторы или ожирение предрасполагают женщин к СПКЯ [13]. Однако метаболическая дисфункция возникает преимущественно у женщин с СПКЯ с диагнозом гиперандрогенизм и овуляторной дисфункцией, независимо от индекса массы тела $[14,15]$. Хотя эти исследования предполагают, что гиперандрогения коррелирует с нарушением регуляции метаболизма, непонятно, почему женщины с СПКЯ становятся инсулинорезистентными и почему у них более высокая частота инсулинорезистентности, чем прогнозируется их индексом массы тела. Инсулинорезистентность и гиперинсулинемия могут способствовать метаболической дисрегуляции за счет усиления выработки андрогенов яичниками и повышения биоактивности андрогенов за счет снижения выработки глобулинов, связывающих половые гормоны [16]. Кроме того, ожирение усугубляет инсулинорезистентность, гиперинсулинемию и выработку андрогенов у женщин с СПКЯ [17], что приводит к порочному кругу гиперандрогении и инсулинорезистентности.

Кишечная и влагалищная микробиота играют весомую роль в патогенезе СПКЯ, а состояния СПКЯ также приводят к изменениям состава микробиоты. Во многих исследованиях сообщалось об изменениях кишечных микроорганизмов у женщин с СПКЯ [18]. Многочисленные доказательства привели к тому, что может существовать связь между микробиомой кишечника и метаболическими, репродуктивными проявлениями СПКЯ.

Кишечная микробиота. Микрофлора кишечника это симбиотические микроорганизмы, обитающие в кишечном тракте человека. По размеру флоры микробиоту кишечника можно разделить на преобладающую и субдоминантную микрофлору. Первый относится к бактериям с высокой плотностью популяции, обычно выше 10-10 KOE / г, в основном включая облигатные, анаэробные бактерии, такие как Bacteroides, Eubacterium, Bifidobacterium, Ruminococcus и Clostridium. В то время как субдоминантная микрофлора состоит из аэробных или факультативных анаэробных бактерий. Плотность населения и разнообразие микробиоты наиболее высоки в толстой кишке.

Кишечные микробы во многих отношениях тесно связаны со здоровьем человека. Микроорганизмы кишечника продуцируют короткоцепочечные жирные кислоты (SCFA), которые играют важную роль в иммуномодуляции [19,20], например, в нейтрализации воспаления легких [21]. Изменения в составе кишечных микробов влияют на функцию печени, а хронические заболевания печени часто сопровождаются дисбактериозом кишечных микробов. Следовательно, регулирование кишечных микробов может быть потенциальной терапией заболеваний печени [22]. Микробиота кишечника также играет роль в возрастном сокращении мышц, изменении размера мышц, а также в составе и функции стареющего скелета [23]. Метаболиты, вырабатываемые микробиотой кишечника, такие как желчные кислоты, могут вносить вклад в патогенез и развитие диабета и ожирения $[24,25]$. Что наиболее важно, основная функция кишечных бактерий - помочь хозяину переваривать пищевые субстраты, такие как углеводы, белки, фитохимические вещества и тому подобное. Эффективное поглощение, 
абсорбция и утилизация метаболитов необходимы для обмена веществ в организме и энергетического обмена [26].

Кроме того, считается, что кишечная микробиота связана с сердечно-сосудистыми заболеваниями, раком, когнитивными функциями и гинекопатией, такими как гиперплазия эндометрия, эндометриоз, СПКЯ и бесплодие, регулируя уровни циркулирующих эстрогенов [27]. В заключение следует отметить, что микробиом кишечника вносит значительный вклад в поражение различных органов или тканей и играет важную роль в нормальном функционировании организма.

Влагалищная микробиота является динамичным и часто критическим фактором в этом патогенном взаимодействии, поскольку изменения характеристик влагалищной микробиоты приводят к потере обычно защищающих Lactobacillus spp. увеличивая тем самым риск нарушений репродуктивной функции. Эти изменения могут быть результатом дефицита эстрогена, противомикробной терапии, контрацептивов или других причин. Во влагалище микробы существуют в тонко настроенных мутуалистических отношениях с хозяином и обеспечивают первую линию защиты от колонизации условно-патогенными микроорганизмами. На протяжении всей жизни женщины микробиота влагалища претерпевает серьезные изменения, связанные с переходными репродуктивными периодами, такими как половое созревание и менопауза [28,29]. В эти периоды микробиота влагалища может влиять на репродуктивную физиологию хозяина, но также может зависеть от физиологии хозяина.

Предполагается несколько путей, с помощью которых некоторые конкретные виды связаны с фенотипами СПКЯ, но конкретный механизм взаимодействия микробов с СПКЯ остается неуловимым, что ограничивает поиск потенциальных методов лечения.

1. Изменения микробиома кишечника у женщин с СПКЯ. Взаимосвязь между СПКЯ и изменениями микробиоты кишечника в последние годы была предметом многочисленных исследований, которые показали значительную разницу в составе микробиома кишечника между пациентами с СПКЯ и здоровыми людьми из контрольной группы [30,31]. Разнообразие а и $\beta$ определяет изменения микробиома: разнообразие альфа (a) рассматривается как показатель здоровья экосистемы и указывает количество видов, присутствующих в сообществе, которые занимают данную среду в конкретном сообществе, тогда как бета ( $\beta$ ) разнообразие показывает, насколько одно сообщество похоже на другое [32,33,34]. В нескольких исследованиях сообщалось об изменении а и $\beta$ разнообразия у пациентов с СПКЯ [35]. В допол- нение к изменению общего состава микробиома те же исследования показали, что при СПкЯ также наблюдается изменение баланса некоторых видов бактерий [36]. Полезные бактерии, такие как лактобациллы и бифидобактерии., которые повышают иммунитет и всасывание питательных веществ, вместо этого значительно снижаются у пациентов с СПКЯ [37]. Модификации кишечной микробиоты при СПкЯ различны, иногда спорны и еще не полностью изучены. Однако в нескольких исследованиях была предпринята попытка изучить взаимосвязь между кишечной микробиотой и СПКЯ; большинство из них было сосредоточено на связи кишечных бактерий с инсулинорезистентностью и половыми гормонами.

2. Микробиом кишечника и инсулинорезистентность. Инсулинорезистентность (ИР) вместе с ожирением тесно связана с микробиомом кишечника, механизмы, которые связывают ИР, микробиом кишечника и СПКЯ, разнообразны [38]. Дисбаланс микробиоты кишечника, повышающий проницаемость кишечника, может определять хроническое воспаление слабой степени за счет активации иммунной системы. Провоспалительные цитокины нарушают функцию рецептора инсулина, вызывая ИР / гиперинсулинемию; действительно, было установлено, что с повышенной проницаемостью кишечника и последующим введением ЛПС в кровообращение мышей и людей повышаются уровни глюкозы в крови и инсулина натощак $[39,40]$. Другая возможная связь между микробиомом кишечника и ИР включает желудочно-кишечные гормоны, такие как грелин и пептид, оба демонстрируют отрицательную корреляцию с ИР и ИМТ $[20,41]$. Однако не во всех исследованиях были обнаружены различия в уровнях грелина и пептида у женщин с СПКЯ по сравнению со здоровыми женщинами, хотя микробиота кишечника может вызывать изменение секреции этих гормонов, что приводит к инсулинорезистентности и гиперинсулинемии $[42,43]$. Взаимосвязь между микробиотой кишечника, медиаторами оси мозг-кишечник и фенотипами СПКЯ еще недостаточно изучена. На сегодняшний день широко признано, что существует связь между ИР у пациентов с СПКЯ и дисбактериозом кишечника. Действительно, как упоминалось выше, состав микробиома кишечника у пациентов с СПкЯ значительно изменен по сравнению с таковым у женщин без СПКЯ. Изменения кишечной микробиоты гораздо более выражены при формах СПКЯ с ИР, которые имеют самый высокий уровень Bacteroidaceae.и большее снижение Prevotellaceae по сравнению с женщинами с СПКЯ без ИР. Более того, пациенты с ИР показали значительную разницу в количестве 
Ruminococcaceae $и$ Lachnospiraceae по сравнению с пациентами, чувствительными к инсулину.

3. Микробиом кишечника и половые гормоны. Существует множество доказательств того, что пол влияет на состав микробиома кишечника. Действительно, микробиом женщин, по сравнению с микробиомом мужчин, характеризуется большим а-разнообразием и относительно меньшим представительством Bacteroides [44]. Были выделены и другие многочисленные различия в микробиоте обоих полов. Prevotella имеет сильную положительную корреляцию с тестостероном и отрицательную связь с концентрацией эстрадиола, поэтому у мужчин их было больше, чем у женщин [45]. Более того, у мужчин было более низкое содержание Clostridia, Methanobrevibacter и Desulfovibrio по сравнению с женщинами [46]. Эти различия могут быть вызваны половыми хромосомами или половыми гормонами; в то время как первый не был описан, последний был тщательно изучен в исследованиях на крысах, которые подтвердили влияние кастрации на микробиом кишечника $[47,48]$. Половые гормоны могут определять изменения микробиома кишечника, активируя рецепторы, присутствующие в желудочно-кишечном тракте, изменяя активность бета-глюкуронидазы или модулируя системный или кишечный иммунитет. Таким образом, была постулирована связь, возможно причинная, между гиперандрогенизмом СПКЯ и изменениями микробиома кишечника. Кроме того, секреция андрогенов и последующая гиперандрогенемия, вызванная ИР, способна стимулировать разложение висцеральной жировой ткани, что приводит к увеличению свободных жирных кислот, что еще больше увеличивает уровни ИР, создавая порочный круг, способствующий возникновению и развитию СПКЯ [49]. Тем не менее, четкая корреляция между гиперандрогенизмом и микробиомом кишечника в определении генеза СПКЯ отсутствует.

4. Микробиом нижних отделов половых путей у пациентов с СПКя. Менопауза, половые гормоны (особенно эстроген), возраст и гигиенические привычки могут влиять на состав микробиома нижних половых путей (НПП), поскольку женщины в препубертатном и постменопаузальном возрасте представляют разные виды микробов. Основными факторами, ведущими к изменению микробиома НПП у пациенток с СПКЯ, являются нерегулярные менструации и аномальные уровни гормонов [50]. Мы знаем, что во время нормального менструального цикла эстроген и прогестерон вызывают периодические изменения в эпидермальных клетках репродуктивного тракта, которые могут играть решающую роль в поддер- жании микросреды НПП, и, учитывая нерегулярность менструального цикла у пациенток с СПКЯ, это может сильно изменить состав микробиомов НПП пациентов с СПКЯ. Микробиом влагалища и матки претерпевает небольшие изменения между двумя фазами менструального цикла (пролиферативная и секреторная фаза): пролиферативный период, по-видимому, связан с увеличением бактериальной пролиферации во влагалище и эндометрии, что может быть причиной того, что микроорганизм в половые пути больных СПКЯ легче изменить. Существенная разница в количестве таксонов между СПКЯ и здоровыми женщинами в микробиомах влагалища и цервикального канала была продемонстрирована секвенированием гена 16S рPHК в общей сложности на 194 микробных образцах. В частности, наблюдалась большая неоднородность состава микробиома как во влагалище, так и в цервикальном канале со значительно сниженной долей Lactobacillus и, наоборот, увеличением нескольких потенциальных патогенных таксонов, таких как Gardnerellavaginalis, Chlamydiatrachomatis и Prevotella., у пациентов с СПКЯ. Более чем один автор обнаружил тесную связь между началом вагиноза, бесплодием, абортом, мертворождением, преждевременными родами, повторной неудачей имплантации и многими другими неблагоприятными исходами беременности, а также снижением уровня Lactobacillus spp. в женских половых путях [51]. Сообщалось, что виды Gardnerella и Prevotella тесно связаны с бактериальным вагинозом (БВ), который часто упускается из виду и может быть трудно лечить изза легких рецидивов и повышения восприимчивости женщины к другим типам инфекций, включая ВИЧ [52]. Gardnerellavaginalis также может быть обнаружена в эндометрии примерно у половины женщин с БВ и может оказывать неблагоприятное воздействие на процедуру имплантации эмбриона и даже на рост плода. Учитывая, что женщины с СПКЯ часто страдают от бесплодия, абортов, остановки плода, преждевременных родов и ряда других неблагоприятных репродуктивных исходов, состав микроорганизмов в НПП женщин с СПКЯ может привести к этим неблагоприятным репродуктивным фенотипам.

5. Метаболиты, связанные с дисбактериозом кишечника и СПкя Хотя ассоциация не является причинно-следственной связью, изучение профилей метаболитов у женщин с СПКЯ по сравнению со здоровыми контрольными женщинами может дать представление о взаимодействиях хозяин / микроб, опосредованных метаболитами, которые могут влиять на патологию СПКЯ. Раннее исследование на женщинах показало, что повышенный 
уровень лактата в сыворотке крови связан с СПкЯ [53], а лактат, продуцируемый хозяином, проникает в просвет кишечника и служит субстратом для бактерий, утилизирующих лактат, потенциально оказывая избирательное давление в микросреде кишечника. Более того, 2 исследования сообщили о связи между повышенным уровнем N-оксида триметиламина (ТМАО) в сыворотке крови и СПКЯ у женщин. N-оксид триметиламина - это печеночный метаболит, который происходит из триметиламина, который продуцируется кишечными микробами из пищевых предшественников. Повышенные уровни ТМАО связаны с повышенным риском сердечно-сосудистых заболеваний. Хотя измерение метаболитов, связанных с микробиотой, в системном кровотоке у женщин с СПкЯ может помочь выявить механизмы регуляции физиологии хозяина кишечными бактериями, исследование изменений метаболитов в кишечнике или кале (в качестве заместителя) также может выявить регуляторные механизмы. В соответствии с этим, 1 исследование модели индуцированного летрозолом СПкЯ на крысах показало значительное снижение фекальных короткоцепочечных жирных кислот, которые продуцируются кишечной бактериальной ферментацией клетчатки и служат сигнальными молекулами в организме хозяина.

Взаимосвязь между желчными кислотами, кишечной микробиотой и метаболическими заболеваниями подчеркивает растущую ключевую роль желчных кислот в регуляции метаболических заболеваний, включая, СПКЯ. Первичные желчные кислоты служат субстратом для микробных ферментов кишечника, которые приводят к образованию вторичных желчных кислот, которые рециркулируют между кишечником и печенью посредством энтерогепатической циркуляции [54]. Исследование глико- и тауро-конъюгированных первичных желчных кислот в системном кровотоке показало, что они были на более высоком уровне у женщин с СПКЯ, чем у здоровых женщин, и были положительно связаны с ГК. С другой стороны, другое исследование показало, что уровень гликохолевой кислоты в сыворотке крови был ниже у женщин с СПКЯ, чем у женщин без заболевания. Кроме того, целенаправленная метаболомика показала, что вторичные желчные кислоты, гликодеоксихолевая кислота (ГДХК) и тауроурсодезоксихолевая кислота (ТУДХК) были ниже в сыворотке и кале женщин с СПКЯ и нормальным весом по сравнению со здоровыми женщинами. Интересно, что ТУДХК, как сообщается, снижается у мышей, получавших трансплантацию фекального микробиома от женщин с СПКЯ или трансплантацией B.Vulgatus, предполагая, что измененной микробиоты кишечника было достаточно, чтобы вызвать изменения в конкретных уровнях желчных кислот. Помимо своей функции по абсорбции липидов, желчные кислоты действуют как сигнальные молекулы, связывая и активируя рецепторы, такие как фарнезоидный рецептор, рецептор витамина $D$, рецептор прегнана $X$, рецептор сфингозин-1-фосфата 2 и мускариновый рецептор М2. Это предполагает роль передачи сигналов желчной кислоты через рецептор фарнезоида Х в регуляции метаболизма. Однако вклад определенных желчных кислот и кишечных бактерий, деконъюгирующих желчные кислоты (например, Bacteroides), в СПКЯ остается неясным.

С лучшим пониманием роли микробиома в патогенезе СПКЯ были предприняты большие усилия для разработки новых вариантов лечения для лечения СПКЯ [55]. Литература была посвящена пробиотикам, пребиотикам и симбиотикам, а также более инновационным методам трансплантации фекальной микробиоты.

\section{Пробиотики, пребиотики и синбиотики}

По данным Всемирной организации здравоохранения (ВОЗ), пробиотики - это «живые микроорганизмы, которые при введении в адекватных количествах приносят пользу здоровью хозяина». Пробиотические микроорганизмы естественным образом содержатся в ферментированных продуктах: они обладают антиоксидантным, антимикробным, противовоспалительным действием, улучшают метаболические параметры, модулируют микробиоту кишечника и регулируют иммунную систему. Применение пробиотиков в качестве терапии подразумевает соответствующее добавление пробиотиков для лечения заболеваний.

Хотя основной механизм остается неясным, терапия пробиотиками показала положительный эффект на метаболический профиль у женщин с СПКЯ [56]. Ахмади и др. сообщили, что добавление пробиотиков (L. acidophilus, L. casei и B. bifidum) в течение 12 недель вызывало статистически значимое снижение веса и ИМТ у пациентов с СПКЯ по сравнению с плацебо, с положительным влиянием на гликемию, триглицериды (TG) и холестерин липопротеинов очень низкой плотности (ЛПОНП). Подобные результаты с добавлениемL. casei, L. acidophilus, L. rhamnosus, L. bulgaricus, B. breve, B. longum и Streptococcusthermophiles были описаны у женщин с СПКЯ в течение 8 недель со значительным снижением уровня глюкозы в плазме и сывороточного инсулина [57]. Более того, Rashadetal. обнаружили, что добавление пробиотиков (L. delbruekii и L. fermentum) в течение 12 недель значительно снижает уровни гомеостатической модели оценки инсулинорезистентности с дополнительным улучшением липидного профиля. Пробиотическая терапия C L. acidophilus, L. plantarum, L. fermentum 
и L. gasseri также доказали возможную роль в модуляции воспалительных процессов при введении в течение 12 недель женщинам с СПКЯ [58].

В метаанализе, не обнаружили значительного влияния пробиотических добавок на антропометрические показатели, такие как вес, ИМТ и окружность талии, у пациентов с СПКЯ по сравнению с плацебо; вместо этого они обнаружили значительное влияние на гликемический контроль, с более низкими уровнями инсулина и на метаболизм липидов, за счет снижения уровней ТГ в сыворотке и повышения ЛПВП. Эти данные предполагают, что добавки с пробиотиками могут применяться в качестве дополнительной терапии для лечения СПКЯ. Влияние пробиотической терапии на гормональный фон женщин с СПКЯ не так широко. В недавнем метанализе Шамасби и др. Сообщили о значительном влиянии пробиотиков на контроль гормональных и воспалительных показателей: значительное снижение индекса свободных андрогенов и малоновогодиальдегида (МД), а также увеличение связывания половых гормонов.

Другое исследование показало, что добавление пробиотиков женщинам с СПКЯ в течение 12 недель оказало благотворное влияние на уровни общего тестостерона, но не повлияло на другие метаболические профили. Примечательно, что пробиотики и их дозировки, используемые в исследованиях, широко варьировались, и именно поэтому в будущих исследованиях потребуется стандартизация этих аспектов.

Из пребиотиков наиболее известны фруктоолигосахариды (ФОС), инулин, галактоолигосахариды (ГОС) и лактулоза. Они изменяют состав микробиоты, положительно влияя на здоровье хозяина.

Некоторые исследования показали, что пребиотики положительно влияют на метаболические маркеры и иммуномодулирующие свойства, поскольку они вызывают рост как Bifidobacterium, так и Lactobacillus, что, в свою очередь, приводит к значительному снижению уровня глюкозы в плазме натощак, сывороточного ТГ, общего холестерина и холестерина ЛПнП, а также значительное повышение уровня холестерина ЛПВП [59]. В этом смысле указали, что некоторые пребиотики непосредственно управляют гипергликемией. Эти результаты могут указывать на то, что присутствие пребиотиков, наряду с пробиотиками, улучшает их эффективность в снижении уровня ЛПНП. Более того, пробиотики (или синбиотики) могут снижать уровень триглицеридов (средняя разница (МД) -17,51 мг / дл, 95\% доверительный интервал от $-29,65$ до -5,36). Уровни андрогенов, гирсутизм и нарушения менструального цикла также могут улучшиться за счет постоянного потребления декстрина у женщин с СПКЯ.
Однако необходимы дополнительные исследования для выяснения и сравнения эффективности нескольких штаммов пробиотиков и различных доз пробиотиков, определения надлежащей продолжительности лечения и демонстрации пользы для здоровья пробиотиков, пребиотиков и синбиотиков в отношении клинических исходов при СПКЯ [60].

\section{Зак^ючение}

Данный обзор литературы показало, что микробиом тесно связан с терапевтическими подходами ко многим заболеваниям.

Во-первых, микробиом может влиять на реакцию, на лекарства и терапию; во-вторых, микробиом сам по себе является источником новой терапии.

Дисбактериоз кишечной микробиоты, возникающий при СПКЯ, приводит к уменьшению разнообразия, а изобилие некоторых видов бактерий вызывает метаболические нарушения.

Текущие данные подчеркивают вклад микробиома кишечника в состояния, не связанные с кишечником, и новые исследования продемонстрировали его участие в СПКЯ, развивается дисфункция яичников, наряду с иммунными изменениями и инсулинорезистентностью $[56,57,58,59]$.

На сегодняшний день причинный вклад состава микробиоты кишечника и нарушения вторичного биосинтеза желчных кислот в патогенезе СПКЯ, по-видимому, доказан. В свете этих данных можно полагать, что будущий терапевтический подход к СПКЯ может включать микробиоту кишечника.

Добавление пребиотиков, пробиотиков и синбиотиков женщинам с СПКЯ, по-видимому, улучшает многие биохимические показатели и благотворно влияет, но механизм до сих пор неясен. Необходимы дальнейшие исследования, чтобы установить роль этих агентов в лечении или, возможно, в профилактике СПКЯ.

Также существенную роль играет и экосистема влагалища человека, она представляет собой динамическую среду, в которой микробы могут влиять на физиологию хозяина, но также и в которой физиология хозяина может влиять на состав и функцию микробиоты влагалища. Виды Lactobacillus исторически были связаны со здоровьем влагалища у женщин с СПКЯ из-за прямого и косвенного защитного характера, обеспечиваемого продуктами Lactobacillus, такими как молочная кислота и бактериоцин, среди других, против разложения слизи и ингибирования патогенов. Сообщаемый непо- 
стоянный врожденный иммунный ответ, наблюдаемый y Lactobacillus- подчеркивает необходимость более глубокого функционального понимания взаимодействия между микробиотой влагалища и физиологией хозяина [28].
Будущие проспективные и особенно рандомизированные клинические исследования необходимы для объяснения механизмов, лежащих в основе этой ассоциации, причин дисбиоза кишечной микробиоты и роли кишечной и влагалищной микробиоты в СПКЯ.

\section{ЛИТЕРАТУРА}

1. Teede H.J., Misso M.L., Costello M.F., Dokras A., Laven J., Moran L., Piltonen T., Norman R.J., Andersen M., Azziz R., et al. Recommendations from the international evidence-based guideline for the assessment and management of polycystic ovary syndrome. Hum. Reprod. 2018; Vol.33:1602-1618.

2. Bozdag G., Mumusoglu S., Zengin D., Karabulut E., Yildiz B.0. The prevalence and phenotypic features of polycystic ovary syndrome: A systematic review and metaanalysis. Hum. Reprod. 2016;- Vol.31:2841-2855.

3. Lambrinoudaki I., Armeni E. Cardiovascular Risk in Postmenopausal Women with Polycystic Ovary Syndrome. Curr. Vasc. Pharmacol. 2019;- Vol.17:579-590.

4. Puurunen J., Piltonen T., Morin-Papunen L., Perheentupa A., Järvelä I., Ruokonen A., Tapanainen J.S. Unfavorable Hormonal, Metabolic, and Inflammatory Alterations Persist after Menopause in Women with PCOS. J. Clin. Endocrinol. Metab. 2011;- Vol.96:1827-1834.

5. Della Corte L., La Rosa V.L., Rapisarda A.M.C., Valenti G., Morra I., Boccellino A., Zizolfi B., Santangelo F., De Rosa N., Sapia F., et al. Current evidences and future perspectives on patient-oriented treatments for polycystic ovary syn-drome: An overview. Ital. J. Gynaecol. Obstet. 2018;- Vol.30:2385.

6. Azziz R., Carmina E., Chen Z., Dunaif A., Laven J.S.E., Legro R.S., Lizneva D., Horowtiz-Natterson B., Teede H.J., Yildiz B.0. Polycystic ovary syndrome. Nat. Rev. Dis. Primers. 2016;-Vol.2:16057.

7. Zawadzki J.K., Dunaif A. Diagnostic criteria for polycystic ovary syndrome: Toward a rational approach. In: Dunaif A., Givens J.R., Haseltine F., Merriam G.R., editors. Polycystic Ovary Syndrome. Blackwell Scientific Publications; Oxford, UK: 1992. Vol. 377-384.

8. Chang J., Azziz R., Legro R., Dewailly D., Franks S., Tarlatzis R., Fauser B., Balen A., Bouchard P., Dalgien E., et al. Revised 2003 consensus on diagnostic criteria and long-term health risks related to polycystic ovary syndrome. Fertil. Steril. 2004;- Vol.81:19-25.

9. Giampaolino P., Della Corte L., De Rosa N., Mercorio A., Bruzzese D., Bifulco G. Ovarian volume and PCOS: A controversial issue. Gynecol. Endocrinol. 2017;- Vol.34:229232.

10. Giampaolino P., Morra I., Della Corte L., Sparice S., Di Carlo C., Nappi C., Bifulco G. Serum anti-Mullerian hormone levels after ovarian drilling for the secondline treatment of polycystic ovary syndrome: A pilot-randomized study comparing laparoscopy and transvaginal hydrolaparoscopy. Gynecol. Endocrinol. 2017;Vol.33:26-29.

11. Giampaolino P., De Rosa N., Della Corte L., Morra I., Mercorio A., Nappi C., Bifulco G. Operative transvaginal hydrolaparoscopy improve ovulation rate after clomiphene failure in polycystic ovary syndrome. Gynecol. Endocrinol. 2017;- Vol.34:32-35.

12. Franks S., I McCarthy M., Hardy K. Development of polycystic ovary syndrome: Involvement of genetic and environmental factors. Int. J. Androl. 2006;- Vol.29:278285.

13. Merkin SS, et al. (2017) Environmental determinants of polycystic ovary syndrome. Fertil Steril 106, Vol.16-24

14. Barber TM, et al. (2017) Metabolic characteristics of women with polycystic ovaries and oligo-amenorrhoea but normal androgen levels: implications for the management of polycystic ovary syndrome. Clin Endocrinol (0xf) 66, Vol. 513-517

15. Moghetti $P$, et al. (2017) Divergences in insulin resistance between the different phenotypes of the polycystic ovary syndrome. J Clin Endocrinol Metab 98, E628-E637

16. Barbieri RL, et al. (2018) Insulin stimulates androgen accumulation in incubations of ovarian stroma obtained from women with hyperandrogenism. J Clin Endocrinol Metab 62, Vol.904-910

17. Plymate SR, et al. (2018) Inhibition of sex hormone-binding globulin production in the human hepatoma (Hep G2) cell line by insulin and prolactin. J Clin Endocrinol Metab 67, Vol.460-464

18. Stepto NK, et al. (2018) Women with polycystic ovary syndrome have intrinsic insulin resistance on euglycaemic-hyperinsulaemic clamp. Hum Reprod 28, Vol.777784

19. W. Ratajczak, A. Ryl, A. Mizerski, et al. Immunomodulatory potential of gut microbiome-derived short-chain fatty acids (SCFAs).-Vol. 66 (1) (2019), pp. 1-12

20. L. Lin, J. Zhang «Role of intestinal microbiota and metabolites on gut homeostasis and human diseases»-Vol.18 (1) (2017), p. 2

21. J.P. Mcaleer, J.K. Kolls Contributions of the intestinal microbiome in lung immunity.-Vol. 48 (1) (2018), pp. 39-49

22. C.A. Woodhouse, V.C. Patel, A. Singanayagam, et al. Review article: the gut microbiome as a therapeutic target in the pathogenesis and treatment of chronic liver disease. - Vol.47 (2) (2018), pp. 192-202

23. G.J. Grosicki, R.A. Fielding, M.S. Lustgarten Gut microbiota contribute to age-related changes in skeletal muscle size, composition, and function: biological basis for a gut-muscle axis.-Vol.102 (4) (2018), pp. 433-442

24. W. Aw, S. Fukuda Understanding the role of the gut ecosystem in diabetes mellitus. - Vol. 9 (1) (2018), pp. 5-12

25. H. Liu, C. Hu, X. Zhang, et al. Role of gut microbiota, bile acids and their cross-talk in the effects of bariatric surgery on obesity and type 2 diabetes.-Vol. 9 (1) (2018), pp. $13-20$

26. I. Rowland, G. Gibson, A. Heinken, et al. Gut microbiota functions: metabolism of nutrients and other food components.-Vol. 57 (1) (2018), pp. 1-24

27. J.M. Baker, L. Al-Nakkash, M.M. Herbst-Kralovetz Estrogen-gut microbiome axis: physiological and clinical implications.-Vol.103 (2017), — Vol. $45-53$. 
28. Gajer P, Brotman RM, Bai G, Sakamoto J, Schütte UME, Zhong X, Koenig SSK, Fu L, Ma ZS, Zhou X, Abdo Z, Forney LJ \& Ravel J (2019). Temporal dynamics of the human vaginal microbiota. Sci Transl Med 4. Vol.132,52.

29. Galhardo C.L., Soares J.M., Simões R.S., Haidar M.A., Rodrigues de Lima G. \& Baracat E.C. (2018). Estrogen effects on the vaginal pH, flora and cytology in late postmenopause after a long period without hormone therapy. Clin Exp Obstet Gynecol.-Vol.33, 85-89.

30. Qin J., Li R., Raes J., Arumugam M., Burgdorf K.S., Manichanh C., Nielsen T., Pons N., Levenez F., Yamada T., et al. A human gut microbial gene catalogue established by metagenomic sequencing. Nature. 2017;- Vol.464:59-65.

31. Fangyuan C., Zhiwen L.A.I., Zusen X.U. Analysis of the gut microbial composition in polycystic ovary syndrome with acne. Zigong Matern. Child. Health Hosp. 2019;Vol.35:2246-2251.

32. Zhou L., Ni Z., Cheng W., Yu J., Sun S., Zhai D., Yu C., Cai Z. Characteristic gut microbiota and predicted metabolic functions in women with PCOS. Endocr. Connect. 2020;-Vol.9:63-73.

33. Lindheim L., Bashir M., Münzker J., Trummer C., Zachhuber V., Leber B., Horvath A., Pieber T.R., Gorkiewicz G., Stadlbauer V., et al. Alterations in Gut Microbiome Composition and Barrier Function Are Associated with Reproductive and Metabolic Defects in Women with Polycystic Ovary Syndrome (PCOS): A Pilot Study. PLoS ONE. 2017;- Vol.12.

34. Liu R., Zhang C., Shi Y., Zhang F., Li L., Wang X., Ling Y., Fu H., Dong W., Shen J., et al. Dysbiosis of Gut Microbiota Associated with Clinical Parameters in Polycystic Ovary Syndrome. Front. Microbiol. 2017;- Vol.8:324.

35. Torres P.J., Siakowska M., Banaszewska B., Pawelczyk L., Duleba A.J., Kelley S.T., Thackray V.G. Gut Microbial Diversity in Women With Polycystic Ovary Syndrome Correlates With Hyperandrogenism. J. Clin. Endocrinol. Metab. 2018;- Vol.103:1502-1511

36. Insenser M., Murri M., Del Campo R., Ángeles Martínez-García M., Fernández-Durán E., Escobar-Morreale H.F. Gut Microbiota and the Polycystic Ovary Syndrome: Influence of Sex, Sex Hormones, and Obesity. J. Clin. Endocrinol. Metab. 2018;-Vol.103:2552-2562.

37. Thursby E., Juge N. Introduction to the human gut microbiota. Biochem. J. 2017;-Vol.474:1823-1836.

38. Thackray V.G. Sex, Microbes, and Polycystic Ovary Syndrome. Trends Endocrinol. Metab. 2019;- Vol.30:54-65.

39. Qi X., Yun C., Sun L., Xia J., Wu Q., Wang L., Wang L., Zhang Y., Liang X., Gonzalez F.J., et al. Gut microbiota-bile acid-interleukin-22 axis orchestrates polycystic ovary syndrome. Nat. Med. 2019;- Vol.25:1225-1233.

40. Zeng B., Lai Z., Sun L., Zhang Z., Yang J., Li Z., Lin J., Zhang Z. Structural and functional profiles of the gut microbial community in polycystic ovary syndrome with insulin resistance (IR-PCOS): A pilot study. Res. Microbiol. 2019;- Vol.170:43-52.

41. Sherman S., Sarsour N., Salehi M., Schroering A., Mell B., Joe B., Hill J.W. Prenatal androgen ex-posure causes hypertension and gut microbiota dysbiosis. Gut Microbes. 2018;- Vol.9:400-421.

42. Guo Y., Qi Y., Yang X., Zhao L., Wen S., Liu Y., Tang L. Association between Polycystic Ovary Syndrome and Gut Microbiota. PLoS ONE. 2017;- Vol.11.

43. Jiao N., Baker S.S., Nugent C.A., Tsompana M., Cai L., Wang Y., Buck M.J., Genco R.J., Baker R.D., Zhu R., et al. Gut microbiome may con-tribute to insulin resistance and systemic inflammation in obese rodents: A meta-analysis. Physiol Genomics. 2018;-Vol.50:54-244.

44. Chen T., Long W., Zhang C., Liu S., Zhao L., Hamaker B.R. Fiber-utilizing capacity varies in Prevotella- versus Bacteroides-dominated gut microbiota. Sci. Rep. $2017 ;-$ Vol.7:1-7.

45. Haro C., Rangel-Zúñiga 0.A., Alcalá-Díaz J.F., Gómez-Delgado F., Pérez-Martínez P., Delgado-Lista J., Quintana-Navarro G.M., Landa B.B., Cortés J.A.N., Tena-Sempere M., et al. Intestinal Microbiota Is Influenced by Gender and Body Mass Index. PLOS ONE. 2016;- Vol.11.

46. Harada N., Hanaoka R., Horiuchi H., Kitakaze T., Mitani T., Inui H., Yamaji R. Castration influences intestinal microflora and induces abdominal obesity in high-fat diet-fed mice. Sci. Rep. 2016;- Vol.6:23001.

47. Choi S., Hwang Y.-J., Shin M.-J., Yi H. Difference in the Gut Microbiome between Ovariectomy-Induced Obesity and Diet-Induced Obesity. J. Microbiol. Biotechnol. 2017;27:2228-2236.

48. Barrea L., Marzullo P., Muscogiuri G., Di Somma C., Scacchi M., Orio F., Aimaretti G., Colao A., Savastano S. Source and amount of carbohydrate in the diet and inflammation in women with polycystic ovary syndrome. Nutr. Res. Rev. 2018;-Vol.31:291-301.

49. Wang L., Zhou J., Gober H.-J., Leung W.T., Huang Z., Pan X., Li C., Zhang N., Wang L. Alterations in the intestinal microbiome associated with PCOS affect the clinical phenotype. Biomed. Pharmacother. 2021;-Vol.133:110958.

50. RatajczakW., Rył A., Mizerski A., Walczakiewicz K., Sipak 0., Laszczyńska M. Immunomodulatory potential of gut microbiome-derived short-chain fatty acids (SCFAs) Acta Biochim. Pol. 2019;- Vol.66:1-12.

51. Zhang J., Sun Z., Jiang S., Bai X., Ma C., Peng Q., Chen K., Chang H., Fang T., Zhang H. Probiotic Bifidobacterium lactis V9 Regulates the Secretion of Sex Hormones in Polycystic Ovary Syndrome Patients through the Gut-Brain Axis. mSystems. 2019;-Vol.17-19.

52. Scheiman J., Luber J.M., Chavkin T.A., et al. Meta-omics analysis of elite athletes identifies a performance-enhancing microbe that functions via lactate metabolism. Nat Med. 2019;-Vol.25(7):1104-1109.

53. Gillis C.C., Hughes E.R., Spiga L., et al. Dysbiosis-associated change in host metabolism generates lactate to support salmonella growth. Cell Host Microbe. 2018;Vol.23(1):54-64.

54. Eyupoglu N.D., Caliskan Guzelce E., Acikgoz A., et al. Circulating gut microbiota metabolite trimethylamine N-oxide and oral contraceptive use in polycystic ovary syndrome. Clin Endocrinol (Oxf). 2019;-Vol.91(6):810-815.

55. Huang J., Liu L., Chen C., Gao Y. PCOS without hyperandrogenism is associated with higher plasma trimethylamine N-oxide levels. BMC Endocr Disord. $2020 ;-$ Vol.20(1):1-9.

56. Ahmadi S., Jamilian M., Karamali M., Tajabadi-Ebrahimi M., Jafari P., Taghizadeh M., Memarzadeh M.R., Asemi Z. Probiotic supplementation and the effects on weight loss, glycaemia and lipid profiles in women with polycystic ovary syndrome: A randomized, double-blind, placebo-controlled trial. Hum. Fertil. 2017;Vol.20:254-261. 
57. Rashad N.M., El-Shal A.S., Amin A.I., Soliman M.H. Effects of probiotics supplementation on macrophage migration inhibitory factor and clinical laboratory feature of polycystic ovary syndrome. J. Funct. Foods. 2017;- Vol.36:317-324.

58. Heshmati J., Farsi F., Yosaee S., Razavi M., Rezaeinejad M., Karimie E., Sepidarkish M. The Effects of Probiotics or Synbiotics Supplementation in Women with Polycystic Ovarian Syndrome: A Systematic Review and Meta-Analysis of Randomized Clinical Trials. Probiotics Antimicrob. Proteins. 2019;-Vol.11:1236-1247.

59. Shamasbi S.G., Ghanbari-Homayi S., Mirghafourvand M. The effect of probiotics, prebiotics, and synbiotics on hormonal and inflammatory indices in women with polycystic ovary syndrome: A systematic review and meta-analysis. Eur. J. Nutr. 2019;- Vol.59:433-450.

60. Shamasbi S.G., Dehgan P., Charandabi S.M.-A., Aliasgarzadeh A., Mirghafourvand M. The effect of resistant dextrin as a prebiotic on metabolic parameters and androgen level in women with polycystic ovarian syndrome: A randomized, triple-blind, controlled, clinical trial. Eur. J. Nutr. 2018;- Vol.58:629-640.

( ) Толстова Анастасия Сергеевна ( asyatol97@mail.ru ), Морозов Максим Владимирович ( morozov_mv93@mail.ru),

Родин Олег Васильевич ( rodin2oleg@gmail.com ), Фазилов Никита Александрович ( Fazilovnikita13@gmail.com ),

Кох Андрей Александрович ( koh-andrei63@yandex.ru ), Безжонова Ангелина Александровна ( abezzhonova@mail.ru ).

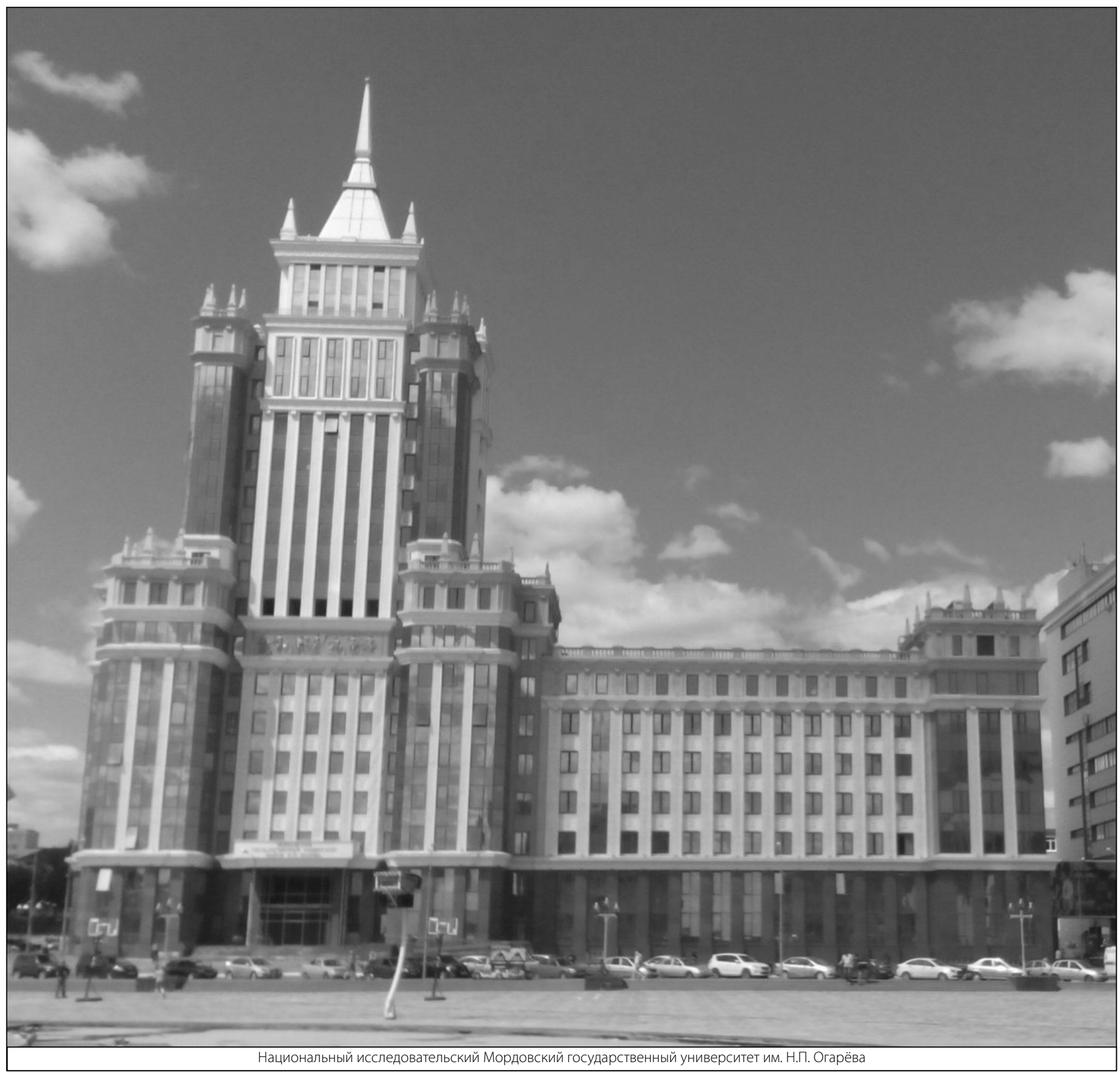

